\title{
TENDÊNCIAS ESPAÇO-TEMPORAIS DAS CHUVAS NA AMAZÔNIA BRASILEIRA
}

Celso Henrique Leite Silva Junior ${ }^{(a)}$, Catherine Torres de Almeida ${ }^{(b)}$, Luiz Eduardo Oliveira e Cruz de Aragão ${ }^{(c)}$, Liana Oighenstein Anderson ${ }^{(d)}$

(a) Mestrado em Sensoriamento Remoto, Instituto Nacional de Pesquisas Espaciais, celso.junior@inpe.br

(b) Doutorado em Sensoriamento Remoto, Instituto Nacional de Pesquisas Espaciais, catherine.almeida@inpe.br

(c) Divisão de Sensoriamento Remoto, Instituto Nacional de Pesquisas Espaciais, laragao@dsr.inpe.br

(d) Centro Nacional de Monitoramento e Alertas de Desastres Naturais, liana.anderson@cemaden.gov.br

\section{Eixo: Climatologia em Diferentes Níveis Escalares: mudanças e variabilidades}

\begin{abstract}
Resumo
Nas últimas décadas profundas mudanças foram observadas na Amazônia, principalmente as relacionadas ao clima. Dessa maneira, o presente artigo apresenta como objetivo uma análise espaçotemporal das tendências na chuva ao longo da Amazônia Legal Brasileira entre os anos de 1998 a 2015. Para isso, foram utilizados dados de chuva derivados do Tropical Rainfall Measuring Mission TRMM, juntamente aos testes não paramétricos Mann-Kendall e Sen's Slope. Foi encontrada redução da quantidade de chuva em anos de ocorrência de secas extremas na região. A análise regional mostrou tendência não-significativa ( $\mathrm{p}$-valor $=0,40)$ de aumento na chuva $\left(6,12 \mathrm{~mm}\right.$ ano ${ }^{-1}$ ). As análises locais evidenciaram que aproximadamente 92,31\% da extensão da Amazônia Legal Brasileira não apresentou tendência. As áreas com tendências significativas de aumento ( $\mathrm{p}$-valor $\leq 0,05$ ) totalizaram cerca de $4,18 \%$ (máximo de $64,92 \mathrm{~mm}^{2}$ ano $^{-1}$ ), enquanto que as áreas com tendências significativas de redução totalizaram cerca de 3,50\% (mínimo de $-49,23 \mathrm{~mm}$ ano ${ }^{-1}$ ). As áreas com tendências de redução ocorreram principalmente em áreas de floresta intacta o que pode trazer impactos negativos ao funcionamento ecológico desses ecossistemas.
\end{abstract}

Palavras chave: Florestas Tropicais, Secas, Mann-Kendall, TRMM, Sensoriamento Remoto.

\section{Introdução}

A Bacia Amazônica abriga mais da metade das florestas tropicais remanescentes do mundo, elas hospedam cerca de 20 a $40 \%$ das espécies de fauna e flora do planeta (LAURANCE; VASCONCELOS, 2009; MAUÉS; OLIVEIRA, 2010).

Nas últimas décadas, importantes mudanças no clima da Amazônia têm ocorrido devido às variações climáticas globais decorrentes de causas naturais ou antrópicas, além das mudanças no microclima local relacionadas às alterações do uso da terra na região (NOBRE; SAMPAIO; SALAZAR, 2007). Tudo isso tem intensificado a ocorrência de secas e cheias extremas na região (MARENGO; ESPINOZA, 2016).

No século passado, oito secas ocorreram na região amazônica com intervalo médio de 12 anos entre elas, e somente nos 16 primeiros anos do século XXI já foram identificadas quatro secas com intervalo médio de 
4 anos entre secas (MARENGO; ESPINOZA, 2016). Essas evidências confirmam os efeitos das mudanças climáticas nas frequências de secas na Amazônia, que foram previstos em modelos globais para o século XXI (MALHI et al., 2008).

Nesse contexto, vários autores encontraram evidências de tendência de redução e aumento na precipitação em estações meteorológicas ao longo da Amazônia Brasileira (PAIVA; CLAKE, 1995; MARCUZZO; CARDOSO; FARIA, 2011; SANTOS; LUCIO; SILVA, 2015; ALMEIDA et al., 2016a; MENEZES; FERNANDES, 2016; SILVA et al., 2016).

No entanto, devido às limitações relativas à distribuição da rede de estações nessa região, pouco se sabe a respeito das áreas não cobertas e principalmente nos últimos 18 anos. Dessa maneira, o presente trabalho tem por objetivo, investigar a tendência espaço-temporal das chuvas entre os anos de 1998 a 2015 na Amazônia Legal Brasileira utilizando dados estimados por sensores remotos.

\section{Materiais e Métodos}

\section{1 Área de Estudo}

A Amazônia Legal (Figura 1) abrange mais da metade do território brasileiro $\left(5.217 .423 \mathrm{~km}^{2}\right.$, cerca de $61 \%$ do território). Essa área administrativa foi instituída em 1953 pela Lei Federal $\mathrm{n}^{\circ} 1.806$, onde foram incluídos os estados do Acre, Amapá, Amazonas, Pará, Rondônia, Roraima, Tocantins, Mato Grosso e parte do Maranhão (a oeste do meridiano de $44^{\circ}$ de longitude oeste) (MARTHA JÚNIOR; CONTINI; NAVARRO, 2011).

Essa área é composta principalmente pela Floreta Tropical Amazônica, que segundo a classificação de Köppen, apresenta um clima do tipo "A", típico de florestas tropicais, sem uma estação fria e temperatura mínima média acima de $18{ }^{\circ} \mathrm{C}$, e é subdividida em três regiões de sub climas: equatorial chuvosa (“Af'), Monção tropical (“Am”) e seco e úmido tropical (“Aw”) (BASTOS, 1982). As áreas com intensa atividade de desmatamento concentram-se na região denominada Arco do Desmatamento que faz fronteira com a região de Cerrado (CAÚLA et al., 2015). 


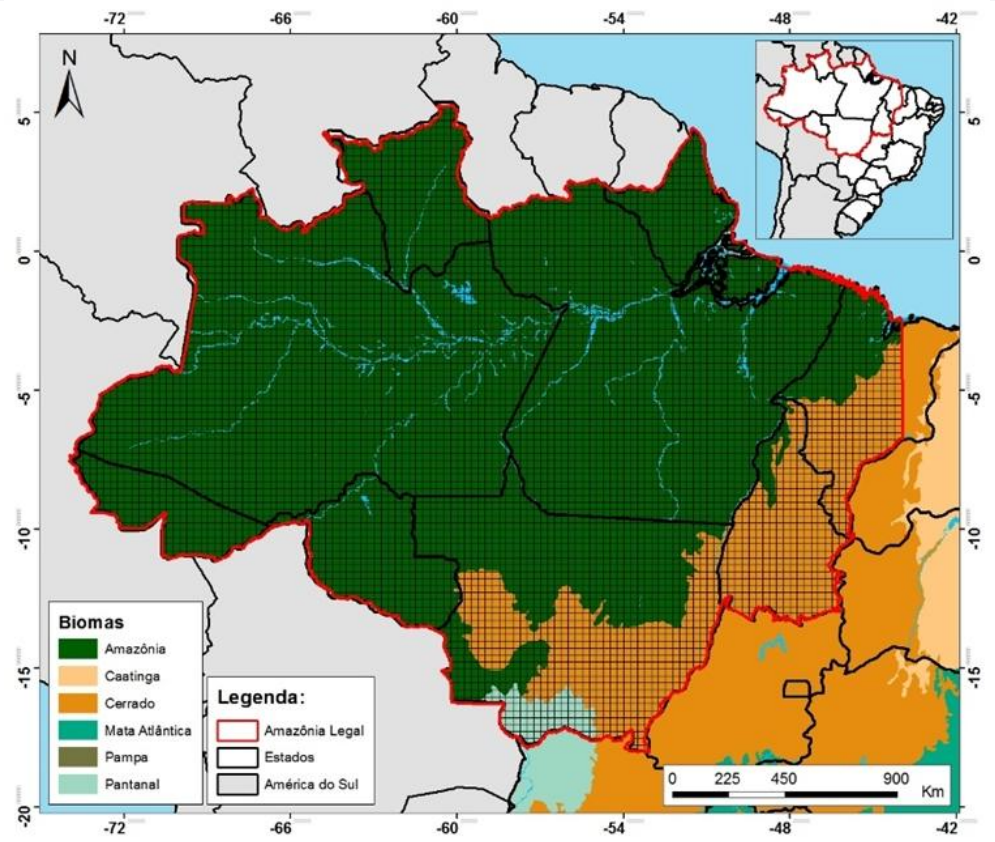

Figura 1 - Localização da área de estudo. A grade na imagem representa os pixels dos dados TRMM.

\subsection{Dados}

Foram utilizados dados mensais de chuva obtidos a partir do produto TRMM 3B43-V7. Esses dados são disponibilizados em uma resolução espacial de $0,25^{\circ}$ por $0,25^{\circ}$ (cerca de 27 por $27 \mathrm{~km}$ ou $729 \mathrm{~km}^{2}$ ), recobrindo o globo terrestre entre as latitudes $50^{\circ} \mathrm{N}$ a $50^{\circ} \mathrm{S}$ (NASA, 2016). Cada pixel nos dados apresentam o valor do acumulado mensal de chuva em milímetros. Esses dados foram validados para a região amazônica, onde foi encontrada boa concordância entre a quantidade de chuva estimada pelo produto e a observada em campo por pluviômetros (ARAGÃO et al., 2007; ALMEIDA et al., 2015; SANTOS et al., 2015).

\subsection{Metodologia}

Os dados mensais de chuva foram compilados em total anual. Em seguida, foi calculada a média de chuva anual por célula. Posteriormente foram calculadas anomalias normalizadas (Equação 1) para investigar o quanto ocorreu de chuva em cada ano em relação à média, normalizada pelo desvio padrão (ARAGÃO et al., 2007).

$$
\text { Anomalia }=\frac{\left(X_{i}-\bar{X}_{1998-2015}\right)}{\sigma_{1998-2015}}
$$




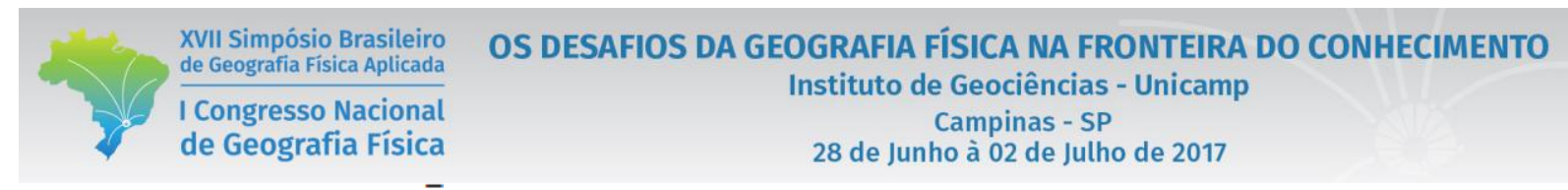

onde $X_{i}$ é o ano em análise, $\bar{X}$ a média da série de 1998 a 2015 e $\sigma$ é o desvio padrão para a mesma série. Foi considerado como significativas (intervalo de confiança de 95\%) as anomalias menores ou igual a 1,96 e maiores ou iguais a 1,96 (Anderson et al., 2010).

Posteriormente foram realizadas uma análise de tendência regional e outra local (pixel a pixel). Na análise regional foi considerado o total de chuva para toda a extensão da Amazônia Legal Brasileira. Para as análises locais foram consideradas as tendências em cada pixel.

A identificação de tendências nas séries temporais foi realizada utilizando o teste estatístico não paramétrico (não pressupõe normalidade nos dados) Mann-Kendall (MANN, 1945; KENDALL, 1975), indicado para avaliação de tendências em séries temporais de dados ambientais (YU; YANG; WU, 2002; HIPEL; MCLEOD, 2005; PORTELA et al., 2011). Adicionalmente foi utilizado o método não paramétrico Sen's Slope que retorna o valor da magnitude na tendência Sen (mudança por unidade de tempo), que é considerado pouco sensível a dados outliers (SEN, 1968). Para as análises foi considerado o nível de significância de $5 \%$ (p-valor $\leq 0,05)$.

Todas as análises foram realizadas no software estatístico R (R CORE TEAM, 2016). Foi utilizado o pacote “Water Quality (wq)”, através da função “mannKen”(JASSBY; CLOERN, 2016).

\section{Resultados e Discussão}

A Figura 2 apresenta as anomalias calculadas para a média por célula do total anual de chuva (análise regional). No ano de 2015 foi observada a maior anomalia negativa significativa de todos os anos analisados (-2,44 desvios padrões), e no ano de 2008 a maior positiva (1,40 desvios padrões), contudo não significativa. Destacam-se ainda as anomalias não-significativas observadas no ano de 1998 (-1,38 desvios padrões) e 2010 (-0,56 desvios padrões).

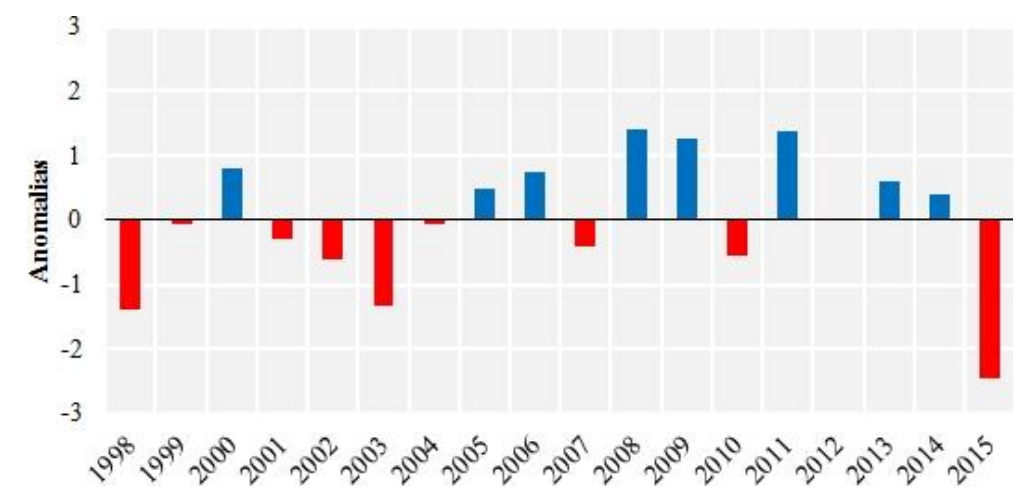

Figura 2 - Anomalias anuais do total de chuva. 
Na Figura 3 é a presentada a distribuição do total anual das chuvas. Entre 1998 a 2015 foi encontrada uma média de 1.655,02( $\pm 117,44) \mathrm{mm}$ de chuva. Foi observado um pico no ano de $2008(1.819,41 \mathrm{~mm})$ e um valor mínimo no ano de 2015 (1.368,36), coincidindo com os anos de maior e menor anomalia, respectivamente.

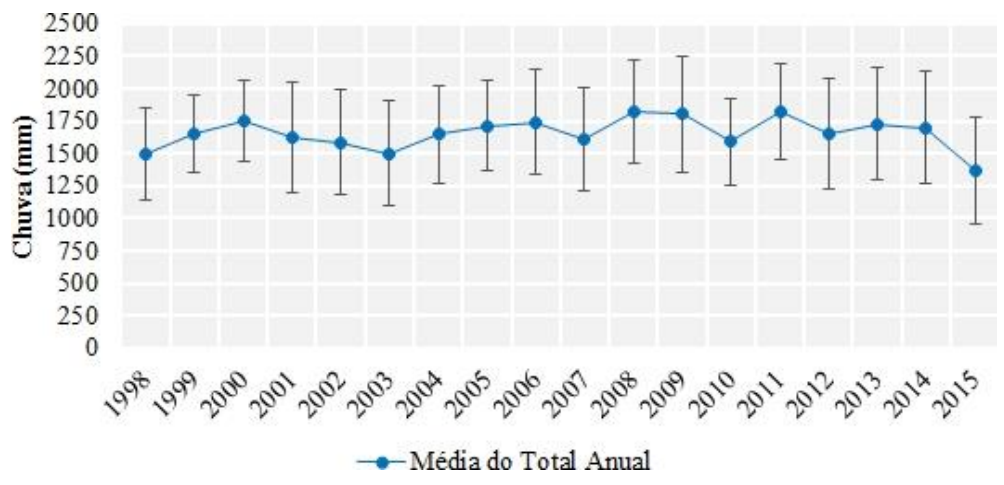

Figura 3 - Distribuição da média anual do total de chuva. Barras em cinza são os desvios padrões.

As anomalias negativas e valores mínimos de chuva observadas nos anos de 1998 (ARAGÃO et al., 2007), 2010 (LEWIS et al., 2011) e 2015 (JIMÉNEZ-MUÑOZ et al., 2016) podem estar associados a ocorrência de secas extremas nos mesmos anos na região, que foram causadas pelos fenômenos El Nino e Oscilação do Atlântico Norte (NAO - North Atlantic Oscillation). A ocorrência de fenômenos de redução na precipitação de chuva induzem o aumento da ocorrência de queimadas, acarretam na mortalidade de arvores, diminuição da biomassa florestal, redução da capacidade da floresta em absorver carbono (NEPSTAD et al., 2004, 2007; ARAGÃO et al., 2007; PHILLIPS et al., 2009; SILVA JUNIOR et al., 2015, 2016; FELDPAUSCH et al., 2016).

Nesse contexto, o teste de Mann-Kendall mostrou uma tendência regional de aumento $(S=0,23)$, no entanto, não significativa (p-valor $=0,40$ ) a uma taxa de crescimento (Sen Slope) de 6,12 mm ano ${ }^{-1}$. Almeida et al. (2016a), por exemplo, demostrou uma tendência de redução não significativas nas chuvas entre 1973 a 2013 para estações meteorológicas da Amazônia Brasileira. Isso indica que regionalmente na Amazônia Legal não existe qualquer tendência na ocorrência das chuvas.

A Figura 4 mostra a média anual de chuva espacializada e as tendências locais significativas ( $\mathrm{p}$-valor $\leq$ 0,05). Foi identificado que a maior média acumulada anual de chuva se concentrou na região norte da Amazônia, com destaque para o Noroeste do estado do Amazonas e Nordeste do estado do Pará. A quantidade média de chuva variou entre $1.039,00 \mathrm{~mm}$ e $3.919,00 \mathrm{~mm}$.

Quanto a distribuição espacial das tendências, foi observado aumento nas chuvas a uma taxa de até 64,92 $\mathrm{mm}$ ano $^{-1}$, concentradas principalmente ao norte do estado de Roraima, sul do Amazonas, norte de Rondônia e norte do estado do Maranhão. Quanto as tendências negativas foram identificadas taxas de até 
$-49,23 \mathrm{~mm}$ ano ${ }^{-1}$, concentradas no centro da Amazônia (principalmente no sudoeste do estado do Pará), oeste da Amazônia (principalmente a oeste e sudoeste do estado do Amazonas e leste do estado do Pará) e a oeste e sudoeste do estado do Amazonas, além do leste do estado do Acre.
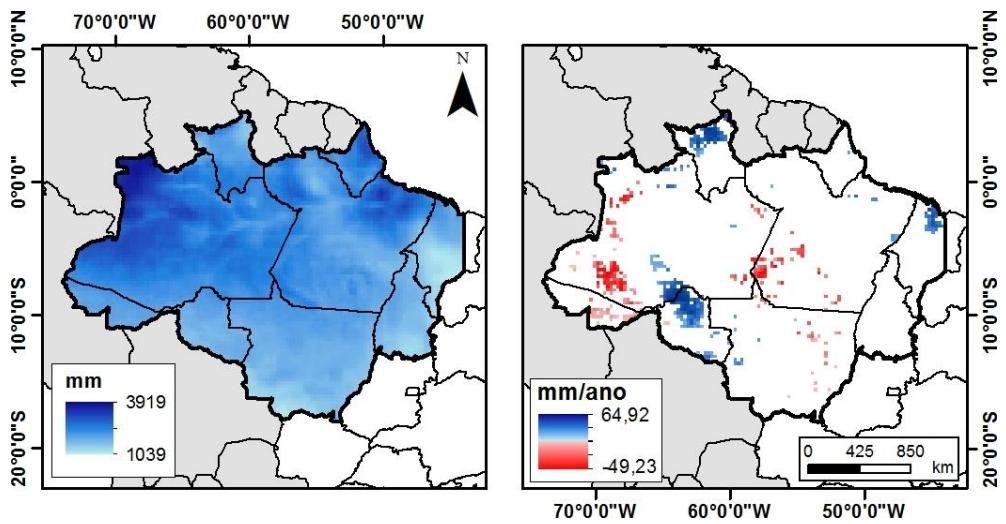

Figura 4 - Média espacializada das chuvas (esquerda). Tendências locais significativas (direita).

As tendências locais de redução anual nas chuvas encontradas para o estado do Acre, corroboram com as análises realizadas por Moreira e Naghettini (2016), onde encontraram tendência negativa em duas das três estações meteorológicas do estado entre os anos de 1971 e 2012, e por Duarte (2005) que ao investigar o comportamento da precipitação pluviométrica entre 1970 a 2000 na cidade de Rio Branco $\mathrm{AC}$ encontrou acentuada tendência de redução. Isso demonstra que a tanto a longo, quanto a curto prazo nesse estado existe uma significativa redução na ocorrência das chuvas.

No estado do Amazonas, Santos, Melo e Brito (2016) encontraram tendências significativa e não significativa de redução do total anual de chuva em 8 estações meteorológicas do estado entre os anos de 1970 e 2010, corroborando com os resultados aqui apresentados. Duas das estações analisadas com tendência negativas se sobrepõe às áreas de tendências significativa de redução encontradas no presente trabalho. Ou seja, a longo e a curto prazo essas áreas apresentam redução significativa nas chuvas.

Para o estado de Rondônia as áreas encontradas com aumento significativo anual corroboram com os resultados encontrados por Furlan (2009) entre os anos de 1961 e 2007. No entanto, a análise conduzida por Bezerra, Dantas e Trindade (2010) entre os anos de 1945 a 2003 não mostraram qualquer tendência na precipitação pluviométrica na região. Isso evidencia que as mudanças nos padrões de chuva nesse Estado podem estar ocorrendo recentemente.

No estado do Mato Grosso, que é uma conhecida fronteira de intensas atividades agrícolas e pecuárias na Amazônia (ALMEIDA et al., 2016b), os resultados evidenciaram que grade parte de seu território não apresentou tendência nas chuvas. No entanto, pequenas áreas difusas, com tendências de redução foram observadas. Marcuzzo, Cardoso e Faria (2011), ao analisar a tendência mensal de precipitação 
pluviométrica em 37 estações na porção amazônica do estado do Mato Grosso entre os anos de 1977 a 2006, contataram tendência de redução na precipitação pluviométrica em 10 meses do ano. Esse fato pode indicar que a inexistência de tendências, possa ser um fenômeno recente nessa região.

No estado do Pará, foram observadas áreas majoritariamente com tendência de redução nas chuvas, principalmente na região sudoeste da região. A análise conduzida por Ribeiro et al. (2014) no município de Tucuruí - PA (região leste do estado) entre ao anos de 1972 a 2012, mostraram não haver tendências nas chuvas na região. Dessa maneira, existe evidencias que tanto a longo prazo, quanto a curto prazo, na região leste do estado não existe tendência nas chuvas.

No estado do Maranhão foram observadas significativas tendências positivas ao norte do estado, principalmente na região conhecida como Baixada Maranhense. Essa região apresenta um complexo sistema de áreas periodicamente alagadas que dependem fortemente da precipitação pluviométrica para a sua manutenção (MENDES et al., 2015; SILVA JUNIOR et al., 2016), em que alterações bruscas, podem causar impactos negativos a esse ambiente e à economia da região (FREIRE et al., 2014), pois o cultivo de arroz em sistema de vazante é importante para a região (FILHO; FERRAZ JÚNIOR, 2009). Em contraponto, outros dois estudos mostraram que em estações meteorológicas nessa mesma região, considerando uma série histórica de longo prazo foram observadas tendência de redução da precipitação pluviométrica (SILVA et al., 2014, 2016), mostrando dessa maneira, indícios que o aumento da precipitação vem acontecendo nas últimas duas décadas na região da Baixada Maranhense.

De forma geral, as áreas sem tendência ou com tendência não significativa (p-valor > 0,05) totalizaram cerca de 92,31\% da área total da Amazônia Legal. As áreas com tendência positivas totalizaram cerca de $4,18 \%$ e as negativas cerca de $3,50 \%$.

As tendências aqui observadas podem ser resultados das mudanças climáticas globais, assim como, resultado do aumentado na frequência de eventos extremos na região (MARENGO; ESPINOZA, 2016). Mudanças no microclima da região devido ao aumento do desmatamento e do lançamento de aerossóis resultantes de queimadas podem também contribuir para essas tendências (LIN et al., 2006; GONÇALVES; MACHADO; KIRSTETTER, 2015; SPRACKLEN; GARCIA-CARRERAS, 2015).

\section{Considerações Finais}

Os resultados aqui apresentados sugerem evidências de alterações nos padrões das chuvas na região da Amazônia Brasileira nos últimos 18 anos. Em alguns estados da região, foram demostradas evidencias de que tendências nas chuvas estejam ocorrendo mais recentemente. 
As áreas com tendências negativas foram observadas principalmente em área de floresta intacta, o que pode acarretar um impacto adverso na vegetação, visto que a vegetação na Amazônia pode ser sensível às variações nos padrões de chuva (HILKER et al., 2014).

As hipóteses aqui apresentadas fornecem subsídios para a elaboração de novos trabalhos que visem novas análises, aprofundamentos e testes.

\section{Bibliografia}

ALMEIDA, C. T.; OLIVEIRA-JÚNIOR, J. F.; DELGADO, R. C.; CUBO, P.; RAMOS, M. C. Spatiotemporal rainfall and temperature trends throughout the Brazilian Legal Amazon, 1973-2013. International Journal of Climatology, jul. 2016a. Disponível em: 〈http://doi.wiley.com/10.1002/joc.4831〉.

ALMEIDA, C. A. de; COUTINHO, A. C.; ESQUERDO, J. C. D. M.; ADAMI, M.; VENTURIERI, A.; DINIZ, C. G.; DESSAY, N.; DURIEUX, L.; GOMES, A. R. High spatial resolution land use and land cover mapping of the Brazilian Legal Amazon in 2008 using Landsat-5/TM and MODIS data. Acta Amazonica, v. 46, n. 3, p. 291-302, set. 2016b. Disponível em: <http://www.scielo.br/scielo.php?script=sci_arttext\&pid=S0044$59672016000300291 \& \operatorname{lng}=$ en\&nrm=iso\&tlng=en>.

ALMEIDA, C. T. de; DELGADO, R. C.; OLIVEIRA JUNIOR, J. F. de; GOIS, G.; CAVALCANTI, A. S. Avaliação das Estimativas de Precipitação do Produto 3B43-TRMM do Estado do Amazonas. Floresta e Ambiente, v. 22, n. 3, p. 279-286, set. 2015. Disponível em: <http://www.scielo.br/scielo.php?script=sci_arttext\&pid=S2179$80872015000300279 \& \operatorname{lng}=$ pt\&nrm=iso\&tlng=en>.

ANDERSON, L. O.; MALHI, Y.; ARAGÃO, L. E. O. C.; LADLE, R.; ARAI, E.; BARBIER, N.; PHILLIPS, O. Remote sensing detection of droughts in Amazonian forest canopies. New Phytologist, v. 187, n. 3, p. 733-750, ago. 2010. Disponível em: 〈http://www.ncbi.nlm.nih.gov/pubmed/20659255〉. Acesso em: 1 dez. 2014.

ARAGÃO, L. E. O. C.; MALHI, Y.; ROMAN-CUESTA, R. M.; SAATCHI, S.; ANDERSON, L. O.; SHIMABUKURO, Y. E. Spatial patterns and fire response of recent Amazonian droughts. Geophysical Research Letters, v. 34, n. 7, p. L07701, 3 abr. 2007. Disponível em: 〈http://doi.wiley.com/10.1029/2006GL028946>. Acesso em: 18 ago. 2014

BASTOS, T. X. O clima da amazônia brasileira segundo koppen. Belém: EMBRAPA-CPATU, 1982.

BEZERRA, R. B.; DANTAS, R. T.; TRINDADE, A. G. Caracterização temporal da precipitação pluvial do município de Porto Velho/RO no período de 1945 a 2003. Sociedade \& Natureza (Online), v. 22, n. 3, p. 609-623, dez. 2010. Disponível em: $\quad<\mathrm{http} / / / \mathrm{www}$. scielo.br/scielo.php?script=sci_arttext\&pid=S1982$45132010000300015 \& \operatorname{lng}=\mathrm{pt} \& \mathrm{nrm}=\mathrm{iso} \& \operatorname{lng}=\mathrm{pt}\rangle$.

CAÚlA, R. H.; OLIVEIRA-JÚNIOR, J. F.; LYRA, G. B.; DELGADO, R. C.; HEILBRON FILHO, P. F. L. Overview of fire foci causes and locations in Brazil based on meteorological satellite data from 1998 to 2011. Environmental Earth Sciences, v. 74, n. 2, p. 1497-1508, 18 jul. 2015. Disponível em: <http://link.springer.com/10.1007/s12665-015-4142-z>.

DUARTE, A. F. Variabilidade e tendência das chuvas em Rio Branco, Acre, Brasil. Revista Brasileira de Meteorologia, v. 20, n. 1, p. 37-42, 2005.

FELDPAUSCH, T. R.; PHILLIPS, O. L.; BRIENEN, R. J. W.; GLOOR, E.; LLOYD, J.; LOPEZ-GONZALEZ, G.; MONTEAGUDO-MENDOZA, A.; MALHI, Y.; ALARCÓN, A.; ÁLVAREZ DÁVILA, E.; ALVAREZ-LOAYZA, P.; ANDRADE, A.; ARAGAO, L. E. O. C.; ARROYO, L.; AYMARD C., G. A.; BAKER, T. R.; BARALOTO, C.; BARROSO, J.; BONAL, D.; CASTRO, W.; CHAMA, V.; CHAVE, J.; DOMINGUES, T. F.; FAUSET, S.; GROOT, N.; HONORIO CORONADO, E.; LAURANCE, S.; LAURANCE, W. F.; LEWIS, S. L.; LICONA, J. C.; MARIMON, B. S.; MARIMON-JUNIOR, B. H.; MENDOZA BAUTISTA, C.; NEILL, D. A.; OLIVEIRA, E. A.; OLIVEIRA DOS SANTOS, C.; PALLQUI CAMACHO, N. C.; PARDO-MOLINA, G.; PRIETO, A.; QUESADA, 
C. A.; RAMÍREZ, F.; RAMÍREZ-ANGULO, H.; RÉJOU-MÉCHAIN, M.; RUDAS, A.; SAIZ, G.; SALOMÃO, R. P.; SILVA-ESPEJO, J. E.; SILVEIRA, M.; TER STEEGE, H.; STROPP, J.; TERBORGH, J.; THOMAS-CAESAR, R.; VAN DER HEIJDEN, G. M. F.; VÁSQUEZ MARTINEZ, R.; VILANOVA, E.; VOS, V. A. Amazon forest response to repeated droughts. Global Biogeochemical Cycles, v. 30, n. 7, p. 964-982, 4 jul. 2016. Disponível em: <http://doi.wiley.com/10.1002/2015GB005133>.

FILHO, M. S. F.; FERRAZ JÚNIOR, A. S. de L. A cultura do arroz em sistema de vazante na Baixada Maranhense, periferia do sudeste da Amazônia. Pesquisa Agropecuária Tropical, v. 39, n. 2, p. 82-91, 2009. Disponível em: $<$ https://www.revistas.ufg.br/pat/article/view/4597>.

FREIRE, A.; MENDES, J.; SILVA, F.; SILVA JUNIOR, C.; LIMA NETO, R. O ambiente geológico-pedológico das Planícies Inundáveis do Maranhão e sua fragilidade às ações antrópicas. In: Proceedings of Safety, Health and Environment World Congress, Cubatão. Anais... Cubatão: COPEC, 23 jul. 2014. Disponível em: <http://proceedings.copec.org.br/index.php/shewc/article/view/2373>.

FURLAN, D. N. Variabilidade temporal e espacial das chuvas e do balanço hídrico no estado de rondônia: caracterização e análise de tendência. 2009. Universidade de São Paulo, Piracicaba, 2009.

GONÇALVES, W. A.; MACHADO, L. A. T.; KIRSTETTER, P.-E. Influence of biomass aerosol on precipitation over the Central Amazon: an observational study. Atmospheric Chemistry and Physics, v. 15, n. 12, p. 6789-6800, 18 jun. 2015. Disponível em: <http://www.atmos-chem-phys.net/15/6789/2015/>.

HILKER, T.; LYAPUSTIN, A. I.; TUCKER, C. J.; HALL, F. G.; MYNENI, R. B.; WANG, Y.; BI, J.; MENDES DE MOURA, Y.; SELLERS, P. J. Vegetation dynamics and rainfall sensitivity of the Amazon. Proceedings of the National Academy of Sciences, v. 111, n. 45, p. 16041-16046, 11 nov. 2014. Disponível em: <http://www.pnas.org/lookup/doi/10.1073/pnas.1404870111>.

HIPEL, K. W.; MCLEOD, A. I. Time series modelling of water resources and environmental systems. 1st. ed. [s.l.] Elsevier, 2005.

JASSBY, A. D.; CLOERN, J. E. Package “wq". Disponível em: <https://cran.rproject.org/web/packages/wq/wq.pdf>. Acesso em: 19 maio. 2016.

JIMÉNEZ-MUÑOZ, J. C.; MATTAR, C.; BARICHIVICH, J.; SANTAMARÍA-ARTIGAS, A.; TAKAHASHI, K.; MALHI, Y.; SOBRINO, J. A.; SCHRIER, G. van der. Record-breaking warming and extreme drought in the Amazon rainforest during the course of El Niño 2015-2016. Scientific Reports, v. 6, p. 33130, 8 set. 2016. Disponível em: 〈http://www.nature.com/articles/srep33130>.

KENDALL, M. G. Rank correlation methods. London: Charles Griffin, 1975.

LAURANCE, W. F.; VASCONCELOS, H. L. Consequências ecológicas da fragmentação florestal na amazônia. Oecologia brasiliensis, v. 13, n. 3, p. 434-451, set. 2009. Disponível em: <http://www.ppgecologia.biologia.ufrj.br/oecologia/index.php/oecologiabrasiliensis/article/view/364/328>.

LEWIS, S. L.; BRANDO, P. M.; PHILLIPS, O. L.; VAN DER HEIJDEN, G. M. F.; NEPSTAD, D. The 2010 Amazon Drought. Science, v. 331, n. 6017, p. 554-554, 4 fev. 2011. Disponível em: <http://www.ncbi.nlm.nih.gov/pubmed/21292971>.

LIN, J. C.; MATSUI, T.; PIELKE, R. A.; KUMMEROW, C. Effects of biomass-burning-derived aerosols on precipitation and clouds in the Amazon Basin: a satellite-based empirical study. Journal of Geophysical Research, v. 111, n. D19, p. D19204, 12 out. 2006. Disponível em: 〈http://doi.wiley.com/10.1029/2005JD006884>.

MALHI, Y.; ROBERTS, J. T.; BETTS, R. A.; KILLEEN, T. J.; LI, W.; NOBRE, C. A. Climate Change, Deforestation, and the Fate of the Amazon. Science, v. 319, n. 5860, p. 169-172, 11 jan. 2008. Disponível em: <http://www.sciencemag.org/cgi/doi/10.1126/science.1146961>.

MANN, H. B. Nonparametric Tests Against Trend. Econometrica, v. 13, n. 3, p. 245, jul. 1945. Disponível em: <http://www.jstor.org/stable/1907187?origin=crossref>.

MARCUZZO, F. F. N.; CARDOSO, M. R. D.; FARIA, T. G. Chuvas na Amazônia mato-grossense: análise histórica e tendência futura. Caminhos de Geografia, v. 12, n. 38, p. 65-75, 2011. Disponível em: <http://www.seer.ufu.br/index.php/caminhosdegeografia/article/view/16443/9188>. 


\section{OS DESAFIOS DA GEOGRAFIA FÍSICA NA FRONTEIRA DO CONHECIMENTO \\ Instituto de Geociências - Unicamp \\ Campinas - SP \\ 28 de Junho à 02 de Julho de 2017}

MARENGO, J. A.; ESPINOZA, J. C. Extreme seasonal droughts and floods in Amazonia: causes, trends and impacts. International Journal of Climatology, v. 36, n. 3, p. 1033-1050, mar. 2016. Disponível em: $<$ http://doi.wiley.com/10.1002/joc.4420>.

MARTHA JÚNIOR, G. B.; CONTINI, E.; NAVARRO, Z. Caracterização da amazônia legal e macrotendências do ambiente externo. Brasília: Embrapa Estudos e Capacitação, 2011.

MAUÉS, M. M.; OLIVEIRA, P. E. A. M. Consequências da fragmentação do habitat na ecologia reprodutiva de espécies arbóreas em florestas tropicais, com ênfase na Amazônia. Oecologia Australis, v. 14, n. 1, p. 238-250, mar. 2010. Disponível em: <http://www.oecologiaaustralis.org/ojs/index.php/oa/article/view/oeco.2010.1401.14/377>.

MENDES, J. J.; SILVA, F. B.; GALVÃO, A. T. F.; JUNIOR, C. H. L. S. Geotecnologias aplicadas no mapeamento de áreas inundáveis na Baixada Maranhense. In: Anais do XVII Simpósio Brasileiro de Sensoriamento Remoto, João Pessoa. Anais... João Pessoa: INPE, 2015.

MENEZES, F. P.; FERNANDES, L. L. ANÁLISE DE TENDÊNCIA E VARIABILIDADE DA PRECIPITAÇÃO NO ESTADO DO PARÁ. Enciclopédia Biosfera, v. 13, n. 24, p. 1580-1591, 6 dez. 2016. Disponível em: $\langle\mathrm{http}: / /$ www.conhecer.org.br/enciclop/2016b/sociais/analise de tendencia.pdf $>$.

MOREIRA, J. G. do V.; NAGHETTINI, M. Detecção de Tendências Monotônicas Temporais e Relação com Erros dos Tipos I e II: Estudo de Caso em Séries de Precipitações Diárias Máximas Anuais do Estado do Acre. Revista Brasileira de Meteorologia, v. 31, n. 4, p. 394-402, dez. 2016. Disponível em: <http://www.scielo.br/scielo.php?script=sci_arttext\&pid=S0102-77862016000800394\&lng=pt\&nrm=iso\&tlng=en>.

NASA. The Tropical Rainfall Measuring Mission (TRMM). Disponível em: 〈http://trmm.gsfc.nasa.gov/>. Acesso em: 1 jan. 2016.

NEPSTAD, D. C.; TOHVER, I. M.; RAY, D.; MOUTINHO, P.; CARDINOT, G. MORTALITY OF LARGE TREES AND LIANAS FOLLOWING EXPERIMENTAL DROUGHT IN AN AMAZON FOREST. Ecology, v. 88, n. 9, p. 2259-2269, set. 2007. Disponível em: 〈http://doi.wiley.com/10.1890/06-1046.1〉.

NEPSTAD, D.; LEFEBVRE, P.; LOPES DA SILVA, U.; TOMASELLA, J.; SCHLESINGER, P.; SOLORZANO, L.; MOUTINHO, P.; RAY, D.; GUERREIRA BENITO, J. Amazon drought and its implications for forest flammability and tree growth: a basin-wide analysis. Global Change Biology, v. 10, n. 5, p. 704-717, maio 2004. Disponível em: 〈http://doi.wiley.com/10.1111/j.1529-8817.2003.00772.x>.

NOBRE, C.; SAMPAIO, G.; SALAZAR, L. Mudanças climáticas e Amazônia. Ciência e Cultura, v. 53, p. 6, 2007. Disponível em: $<$ http://cienciaecultura.bvs.br/scielo.php?pid=S000967252007000300012\&script=sci_arttext\&tlng=en>.

PAIVA, E. M. C. D. de; CLAKE, R. T. Análise de Tendencia de Precipitação na Amazônia. Revista Brasileira de Meteorologia, v. 10, n. 1/2, p. 37-41, 1995.

PHILLIPS, O. L.; ARAGAO, L. E. O. C.; LEWIS, S. L.; FISHER, J. B.; LLOYD, J.; LOPEZ-GONZALEZ, G.; MALHI, Y.; MONTEAGUDO, A.; PEACOCK, J.; QUESADA, C. a; VAN DER HEIJDEN, G.; ALMEIDA, S.; AMARAL, I.; ARROYO, L.; AYMARD, G.; BAKER, T. R.; BANKI, O.; BLANC, L.; BONAL, D.; BRANDO, P.; CHAVE, J.; DE OLIVEIRA, A. C. A.; CARDOZO, N. D.; CZIMCZIK, C. I.; FELDPAUSCH, T. R.; FREITAS, M. A.; GLOOR, E.; HIGUCHI, N.; JIMENEZ, E.; LLOYD, G.; MEIR, P.; MENDOZA, C.; MOREL, A.; NEILL, D. A.; NEPSTAD, D.; PATINO, S.; PENUELA, M. C.; PRIETO, A.; RAMIREZ, F.; SCHWARZ, M.; SILVA, J.; SILVEIRA, M.; THOMAS, A. S.; STEEGE, H. Ter; STROPP, J.; VASQUEZ, R.; ZELAZOWSKI, P.; DAVILA, E. A.; ANDELMAN, S.; ANDRADE, A.; CHAO, K.-J.; ERWIN, T.; DI FIORE, A.; C., E. H.; KEELING, H.; KILLEEN, T. J.; LAURANCE, W. F.; CRUZ, A. P.; PITMAN, N. C. A.; VARGAS, P. N.; RAMIREZ-ANGULO, H.; RUDAS, A.; SALAMAO, R.; SILVA, N.; TERBORGH, J.; TORRES-LEZAMA, A. Drought Sensitivity of the Amazon Rainforest. Science, v. 323, n. 5919, p. 1344-1347, 6 mar. 2009. Disponível em: <http://www.sciencemag.org/cgi/doi/10.1126/science.1164033>. Acesso em: 14 jul. 2014.

PORTELA, M. M.; QUINTELA, A. C.; SANTOS, J. F.; VAZ, C.; MARTINS, C. Tendências em séries temporais de variáveis hidrológicas. Recursos Hídricos, v. 32, n. 1, p. 43-60, 2011.

R CORE TEAM. R: A language and environment for statistical computing. Disponível em: <https://www.rproject.org/>. Acesso em: 1 jan. 2016. 


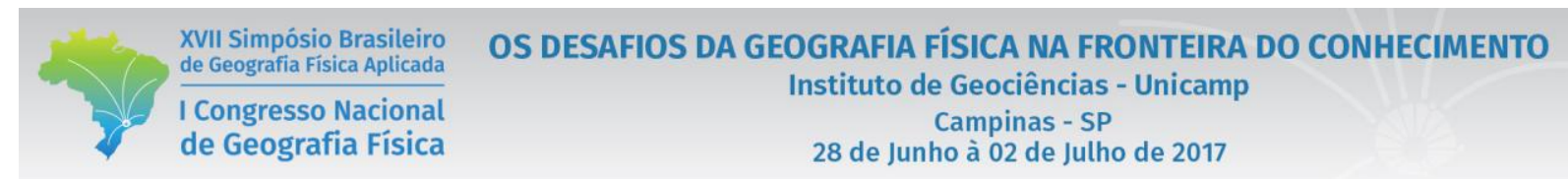

RIBEIRO, R. E. P.; ÁVILA, P. R.; BRITO, J. I.; SANTOS, E. G.; SOUSA, L. F. Análise da Tendência Climática nas Séries Temporais de Temperatura e Precipitação de Tucuruí-Pará. Revista Brasileira de Geografia Física, v. 7 , n. 5, p. 798-807, 2014.

SANTOS, E. B.; LUCIO, P. S.; SILVA, C. M. S. e. Análise de Tendência da Precipitação Diária na Amazônia Brasileira. Revista Brasileira de Geografia Física, v. 8, n. 4, p. 1041-1052, 2015.

SANTOS, J. R. N.; SILVA, F. B.; SILVA JUNIOR, C. H. L.; ARAÚJO, M. L. S. de. Precisão dos dados do satélite Tropical Rainfall Measuring Mission (TRMM) na região de transição Amazônia-Cerrado no Estado do Maranhão. In: XVII Simpósio Brasileiro de Sensoriamento, João Pessoa. Anais... João Pessoa: INPE, 2015.

SANTOS, C. A. C. dos; MELO, M. M. M. S.; BRITO, J. I. B. de. Tendências de Índices de Extremos Climáticos para o Estado do Amazonas e suas Relações com a TSM dos Oceanos Tropicais. Revista Brasileira de Meteorologia, v. 31, n. 1, p. $1-10, \quad$ mar. 2016 . Disponível em: <http://www.scielo.br/scielo.php?script=sci_arttext\&pid=S0102-77862016000100001\&lng=pt\&nrm=iso\&tlng=en>.

SEN, P. K. Estimates of the Regression Coefficient Based on Kendall's Tau. Journal of the American Statistical Association, v. $\quad 63, \quad$ n. $\quad 324, \quad$ p. $1379, \quad$ dez. $1968 . \quad$ Disponível em: <http://www.jstor.org/stable/2285891?origin=crossref $>$.

SILVA, F. B.; SANTOS, J. R. N.; FEITOSA, F. E. C. S.; SILVA, I. D. C.; ARAÚJO, M. L. S. de; GUTERRES, C. E.; SANTOS, J. S. dos; RIBEIRO, C. V.; BEZERRA, D. da S.; NERES, R. L. Evidências de Mudanças Climáticas na Região de Transição Amazônia-Cerrado no Estado do Maranhão. Revista Brasileira de Meteorologia, v. 31, n. 3, p. 330-336, set. 2016. Disponível em: <http://www.scielo.br/scielo.php?script=sci_arttext\&pid=S0102$77862016000700330 \& \operatorname{lng}=$ pt\&nrm=iso\&tlng=en>.

SILVA, F.; SANTOS, J.; ARAÚJO, M.; SILVA JUNIOR, C. ANÁlISE ESPAÇO-TEMPORAL DA PRECIPITAÇÃO NO ESTADO DO MARANHÃO NO PERÍODO DE 2003 A 2012. In: XIV Safety, Health and Environment World Congress, Cubatão. Anais... Cubatão: COPEC, 23 jul. 2014. Disponível em: <http://proceedings.copec.org.br/index.php/shewc/article/view/2375>.

SILVA JUNIOR, C. H. L.; FREIRE, A. T. G.; ANDERSON, L. O.; SILVA, F. B.; MENDES, J. J.; SILVA JUNIOR, C. H. L.; ANDERSON, L. O.; ARAGÃO, L. E. O. E. C. De; SILVA, F. B.; MENDES, J. J. A zona de transição entre a Amazônia e o Cerrado no estado do Maranhão. Parte II: Caracterização preliminar dos dados de área queimada (Produto MODIS MCD45A1). In: XVII Simpósio Brasileiro de SensoriamentoSimpósio Brasileiro de Sensoriamento, João Pessoa. Anais... João Pessoa: INPE, $2015 . \quad$ Disponível em: <http://urlib.net/rep/8JMKD3MGP6W34M/3JM4K59?ibiurl.language=pt-BR>.

SILVA JUNIOR, C. H. L.; FREIRE, A. T. G.; RODRIGUES, T. C. S.; VIEGAS, J. C.; BEZERRA, D. S. Dinâmica das Queimadas na Baixada Maranhense. InterEspaço: Revista de Geografia e Interdisciplinaridade, v. 2, n. 5, p. 355-375, 1 set. 2016. Disponível em: <http://www.bibliotekevirtual.org/index.php/2013-02-07-03-02-35/2013-0207-03-03-11/1933-inter-espaco/v02n05/20077-dinamica-das-queimadas-na-baixada-maranhense.html>.

SPRACKLEN, D. V.; GARCIA-CARRERAS, L. The impact of Amazonian deforestation on Amazon basin rainfall. Geophysical Research Letters, v. 42, n. 21, p. 9546-9552, 16 nov. 2015. Disponível em: <http://doi.wiley.com/10.1002/2015GL066063>.

YU, P.-S.; YANG, T.-C.; WU, C.-K. Impact of climate change on water resources in southern Taiwan. Journal of Hydrology, v. 260, n. 1-4, p. 161-175, mar. 2002. Disponível em: <http://linkinghub.elsevier.com/retrieve/pii/S002216940100614X>. 